Fig. 14. Palpe des Leptus Sambuci bei derselben Vergrösserung. a Nagel. b Vorletztes Glied. c Letztes Glied mit 2 gefiederten und 1 ungefiederien Tastborste.

Fig. 15. Mandibula Lepti Sambuci bei gleicher Vergrösserung.

Fig. 16. Die äussersten Glieder des ersten Fussparas mit 3zehiger Kralle von Leptus Hypudaei, bei 620 facher Vergrösserung.

NB. Sämmtliche Abbildungen sind durch die Chambre claire von 0 berbäuser von mir angefertigt.

\title{
XIX.
}

\section{Beiträge zur Kenntniss der hereditären Knochensyphilis.}

Von W. Waldeyer in Strassburg und H. Köbner in Breslau.

Die interessanten Mittheilungen Wegner's ${ }^{1}$ ), ,über hereditäre Knochensyphilis bei jungen Kindern, " welchen nach einer vorläufigen Notiz von $\mathbf{K} \ddot{b} \mathbf{b n e r ^ { 2 }}$ ) gelegentlich der Section eines an Syphilis e Vaccinatione gestorbenen Kindes auch eine nicht geringe Bedeutung für andere Fragen zuzukommen scheint, haben uns veranlasst, alle neuerdings zu unserer Kenntniss kommenden Fälle von hereditärer Syphilis einer Nachuntersuchung zu unterziehen. Abgesehen davon, dass schon eine einfache Bestätigung der Wegn er'schen Angaben bei der Wichtigkeit des Gegenstandes eine kurze Veröffentlichung unserer Befunde rechtfertigen dürfte, haben wir auch einige Punkte hervorzuheben, welche das bereits Bekannte erweitern und ergänzen.

Unsere Beobachtungen beruhen auf der Autopsie von 12 Fällen. Vorerst können wir vollkommen bestätigen, dass die hereditär syphilitischen Veränderungen der Epiphysengrenzen sich constant vorfanden, dass sich uns bisher kein Ausnahmefall gezeigt hat. Selbst wenn, wie in zwei der unten anzuführenden Beobachtungen (Fall 10 und 12), makroskopisch sichtbare Veränderungen zu fehlen schienen, liessen sich dieselben mikroskopisch noch mit der grössten Sicher heit erkennen.

1) Wegner, Dieses Archiv Bd. L. S. 305 u. ff.

2) Köbner, Die Uebertragung der Syphilis durch die Vaccination. Archiv f. Dermatologie u. Syph. 1871. Hft. 2. S. 133 a. ff. Nachtrag. Ibid. Hft. 4. S.507. 


\section{8}

Weniger sicher trifft man, wie wir Wegner gleichfalls beipfichten, Veränderungen der Schädelknochen und ihrer Adnexa. In einem Falle fanden wir doppelseitige gummöse interne Periostitis an beiden Stirnbeinen mit reichlicher, wie es schien, schubweise erfolgter Extravasatbildung, ähnlich wie bei einer Pachymeningitis chronica haemorhagica; in mehreren anderen Fällen disseminirte, hirsekorn- bis linsengrosse Granulationsknötchen des äusseren Schädelperiostes. Auffallend war uns ferner eine nicht selten vorkommende, aussergewöhnlich derbe und feste Beschaffenheit, sowie eine Verdickung der Dura mater, welche mitunter selbst bei macerirt ausgestossenen syphilitischen Frïchten den Schädelknochen sehr fest adhärirte. Indess constant sind alle diese Veränderungen an den Schädelknochen keineswegs, -

Was die Rothrenknochen betriff, so möchten wir auf die ron Bouchut ${ }^{\text {) }}$ erwähnte grössere Härte derselben kein besonderes Gewicht legen, da Vergleiche mit normalen gleichaltrigen Knochen uns keine bemerkenswerthen Unterschiede ergeben und ferner bei verschiedenen, in Bezug auf das knochensystem normalen Kindern desselben Alters, so weit die gewöhnliche Abschätzungsmethode hier überhaupt ein sicheres Urtheil erlaubt, Schwankungen des Dichtigkeitsgrades ebenfalls vorkommen. Hingegen können wir bezüglich der makroskopischen syphilitischen Veränderungen an den Grenzen der Epipbysen und der Bippenknorpel der Darstellung Wegner's in allen Pankten beipflichten. Wir sahen alle Stadien, von der einfachen, mitunter: nur geringen Verbreitung dex Korpelwucherungszone und der spongioiden Schicht Guérin's an bis zur completen Loslösung der Epiphysen, welche bei einem von Köbner beobachteten vierwöchentlichen Kinde - bei Abwesenheit anderer, für congenitalo Syphilis charakteristischen Zeichen mit Ausnahme etwa von mebrexen miliaren Pusteln an den Fussoulen - bereits intra vitam dingnosticirt werden konnte.

Fall 3, Ein am 28, September 1870 geborenes, ziemlich gut genährtes Mädcher zeigte an 24. October 1870 auf den sichtharen Schleimbäuten und der Haut des gapen körpers nichts Pathologisches ansele drei sehr kleinen Pusteln auf der einen und einer eben Eolchen auf der anderen Planta pedis. Auffallend

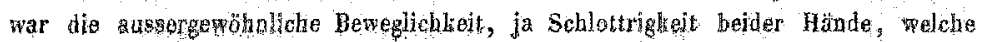
nur auf eine Ablobang der unteren Epiphysen von den Diaphysen der Yorderarm-

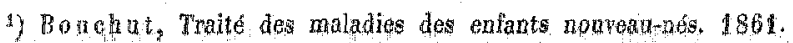


knochen hezogen werden könnte. Nach den Nägeln zu ortheilen, Far das Kind um einige Wochen zu früh geboren. Es war von der, wie sich herausstellt, noch mit Condylomata lata genitalium behafteten Mutter, einem unehelich geschwängerten Mädchen, gesäugt ond noch mit keinerlei Medicament behandelt worden. Die Matter, deren Infectionstermin sich nicht sicher feststellen liess, batfe vom 12. August ej. bis zur Niederkunft Sublimat-Chlornatrium (in Summa etwa 0,33 Grm. Sublimat) gebraucht. - Schon in der folgenden Nacht starb das Kind. Section 25. October. Gehirnödem; Kehlkopf, Trachea, Bronchi normal, mil schaumiger Flüssigkeit bedeckt. In allen Lappen beider L ung en zablreiche, stecknadelkopf- bis fast bohnengrosse, umschriebene, derbe Heerde, durch lufthaltiges Parenchym getrennt; sie wölben die Pleura hervor, welche jedoch intact ist. Die kleineren Heerde sind grauröthlich, die grösseren gelbweiss und von einem rothen, körnigen, infiltrirten Hofe umgeben; die letzteren sind in der Mitte puriform erweicht, ähnlich central gesckmolzenen Käseknoten. Mikroskopiscb zeigt sich eine interstitielle, toberall den Alveoleriwänden, den Gefässen und Bronchien folgende Zellenwacherang mit spindelförmigen und unregelmässig rundlichen Elementen. Die Alveolen durchweg in dieser Wucherung untergegangen, einzelne an der Grenze noch kenntlich an den kleinen Anbäufungen epithelialer grösserer Zellen. Die erweichten centralen Massen bestehen durchweg aus gut erhaltenen rundlichen, Eiterkörperchen ähnlichen Zellen, so dass es sich bier nicht um eine Einschmelzung eines käsigen Productes, sondern um eine rapide eiterähnlicbe Zellenbildung und -Anhäufung im Centrum festerer Granulationstueerde handelt. - In der Leber unter der Serosa mehrere, banfliornbis sechsergrosse hellere Flecke, welche sich scharf von der Umgebung abgrenzen. Mikroskopisch: interstitielle Zellenwucherung zwischen den Leberzellea, namentlich um die Pfortaderäste herum, àhnlich wie bei beginnender Cirrhose. - Die linorpeligen Epiphysen der Humeri und beider Vorderarmknochen; - rechterseits vorgeschrittener als links, an den Humeris stärker an dea oberen, an den Vorderarmknochen stärker an den unteren Enden, - von den Diaphysen gelöst, die Lösung namentlich an den letzteren durch die Haut hindurch zu erkennen. Zwischen Epi- und Diaphysen befindet sich eine röthliche eiteräbnliche Masse, welche mikroskopisch aus Spindelzellen, Rundzellen und einzelnen Myeloplaxes besteht. Die tiefste Knorpelzone an der oberen Humerusepiphyse in der Ausdehnung von etwa 2-3 Mm. bläulich durchscheinend; die darunter gelegene weissgelbliche Zone verkalkten Koorpejgewebes überall etwas dicker, als gewöhnlich, bis etwa $1 \mathrm{Mm}$, nirgends jedoch so stark, als sie von Wegner beschrieben wird. Dann findet sich an den oberen Enden beider Humeri dicht unter der eben genannten Zone, "und bis zu $4 \mathrm{Mm}$. Tiefe in die bereits rerknöcherte spongiöse Substanz der Diaphyse hineinragend, eine pulpöse, elastische, grauröthliche Gewebsmasse von unregelmässig rundlicher Umgrenzung und über Erbsengrösse, welche ohne scharfe Grenze in die Spongiosa übergeht and an diesem Orte deren Stelle einnimmt; bie und da liegt ein einzelnes Knochenspiculum in dieser Masse.

Besonders auffallend sind ferner' $1-4 \mathrm{Mm}$. dicke, noch nicht ganz verknöcherte periostale Koochenbildungen, welche überall dicht unterhalb der Epiphyse der Diaphyse aufgelagert sind, sich jeduch noch leicbt von letzterer trennen lassen. Besonders mächtig erscheint diese Masse, ron einem röthlichen gelatinösen Gewebe 
umgeben, dicht unter der Epiphyse des rechten Bumerus, welche sie wie ein Stalaktitenkranz umgiebt. (Diese Massen gleichen makroskopisch den unrollkommen verknöcherten periostalen Ablagerungen bei der Rachitis). Mikroskopisch bestehen jene weichen Massen aus dicht gedrängten, selı weicken Zellen der verschiedensten Form, am meisten den Zellen einer jungen gummösen Bildung entsprechend.

Es verdient aber besonders herworgehoben zu werden, dass, wie bereits oben bemerkt wurde, die makroskopisch sichtbaren Veränderungen winimal sein ǩönnen, so dass man im Zweifel bleibt, ob sie überhaupt vorhanden sind, während dabei mikroskopisch gan charakteristische Anomalien gefunden werden. Diese Anomalien beruhen nach unseren Erfahrungen wesentlich auf folgenden Punkten:

Zunächst fällt eine vermehrte Wucherung der Knorpelzellen an der Ossificationsgrenze in's Ange. Die Abkömmlinge der Knorpelzellen selbst haben, namentlich in den tieferen, der Ossificationsgrenze näher liegenden Schichten, nicht das gewöhnliche, vom nopmalen Knochen her bekanute Aussehen, sondern nähern sich in Form und Grösse bleinen rundlichen Granulationszellen, wie man sie in syphilitischen Producten anzutreffen pflegt. -

Das unregelmässige záclige Ineinandergreifen der Ossificationszone ard des Knorpels fanden wir ebenso, wie Wegner, und glauben auch grosses Gewicht auf dasselbe zur Charaliterisirung der in Rede stehenden Veränderungen legen zu solien. - -

Ein zweiter, asserst wichtiger Punkt liegt in dem bistologischen Verhalten desjenigen Gewebes, welches die jungen Markräume, sowie die zwischen den Baken der spongioulen Schicht Guérin's liegenden Rälure ausfült. Dasselbe hat nicht den Charalster des normalen Markgewebes, sondern stellt sich an den am meisten veränderten Stellen, um es mit einerm Worte zu sagen, als ein indiferentes, schwer zu charakterisirendes Granulationsgewebe dar, wie wir es bel syphilitischen Veranderungen anderer Organe uberal! antrefien. Wabrend im nomalen Markgewebe Zellen ron Juphatischem Charakter, wholich wie sie in der Niz und den Lymphdrusen vorkmmen, den Hauptbestandbeil ausmachen, daneben häufg auch nielkemige Dlemente (Myelophaxes) won beträchticher Grösse gefunden werden, liegen in den syptilitisch veränderten Bezirken bleire, randioh eckge und spindelige Elemente vor, welche unter einander durch Fortsalze zusammentangen und sich schwer 
durch Pinseln oder Ausschütteln von einander trennen lassen. Das durch Aggregation dieser Zellen erzeugte Gewebe ist bald mehr, bald weniger dicht und macht ganz dieselbe Stufenleiter von Veränderungen durch, wie sie an syphilitischen Producten anderer Orte wahrgenommen werden. Wir finden dort bekanntlich weichere, eiterahnliche Massen von der Consistenz eines Chalazion, dann wieder derb gefiugte Producte, welche durchaus dem Charakter einer gewöhnlichen Neubildung enisprechen, und dazwischen alle nur denkbaren Uebergänge. Dasselbe trifft man bei aufmerksamer Betrachtung an den syphilitisch veränderten Knochengrenzen. Fälle, bei denen die Epiphysen abgelöst erscheinen, zeigen zwischen Epi- und Diaphyse jenes weiche, gallertige, am besten der Consistenz eines Chalazions zu vergleichende Gewebe, das mitunter sogar eiterähnlich erscheinen kann. Doch haben wir uns niemals, selbst in den ausgesprochensten Fällen nicht, von dem Vorbandensein wirklichen Eiters überzeugen können. Wir fanden an feinen Schnitten und frischen Zupfpräparaten dieses Gewebe vascularisirt und die einzelnen Zellen desselben vielfach unter einander durch mehr oder minder entwickelte Fortsătze im Zusammenhange. Es entspricht daher diese Form des Granulationsgewebes, wie es scheint, genau jenen weichen balbflüssigen Gummigewächsen, welche man nicht selten subperiosteal antrifft.

Die festeren syphilitischen epiphysären Neubildungen sind oft ziemlich genau abgegrenat und äbneln mitunter echten Gummigeschwülsten, wie sie in der Leber, im Gehirn sich entwickeln. Auch an den bekannten necrobiotischen Vorgängen, denen die syphilitischen Producte so häufig unterliegen, fehlt es nicht, wie béreits Wegner mitgetheilt bat. Nur sehen wir uns genöthigt, in der Erklärung dieser Necrobiosen von dem Letzteren abzuweichen. Wegner spricht an verschiedenen Stellen seiner Arbeit (S. $311 \mathrm{u}, 314$ ) von einer grossen Armuth an Blutgefässen oder von einem vollkommenen Fehlen derselben in der Zone der präparatorischen Knorpelverkalkung, d. h. der spongioiden Schicht. Wir haben uns dagegen mehrach und besonders an einem, von Dr. Carl Weigert sorglältig injicirten Präparate an vielen, aus der Tibia entnommenen Schnitten mit Sicherheit überzeugt, dass die Blutgefässe keineswegs feblen, sondern in dem ganzen, hier in Betracht kommenden Gebiete, mitunter sogar sehr reichlich auch in der spongioiden Schicht, 
entwickelt sind. Die Necrobiose der, zwischen den jüngsten verkalkten Knorpellagen und den normalen tiefer liegenden partien befindlichen Schicht kann daher nicht auf einen Mangel an Blutgefässen zurückgefübrt werden, sondern ist als ein vor der Hand nicht mit Sicherheit zu erklärender Vorgang in derselben Weise aufzufassen, wie die so häufgen Necrobiosen sypbilitischer Producte überhaupt. Wir können hier nur an den, von Biesiadecki a. A. für den Zerfall syphilitischer Producte hervorgehobenen Erklärungsgrund erinnern, der aller Wahrscheinlichkeit nach auch hier zutreffend ist. Es ist bekannt, dass dieselben sich vorzugsweise in und längs der Adventitien der Gefässe auszubreiten phegen, wie dies von verschiedenen Seiten beschrieben und auch yon ans, namentlich an Hautsypbiliden, constant gesehen worden ist. Unläugbar muss, worauf Biesiadeck i auch das Hauptgewicht gelegt hat, durch diese oft beträchtlichen Zellenwucherungen das Lumen der Gefâsse beengt und die Circulation beeinträchtigt werden. Auf diese Weise können offenbar die Necrobiosen syphilitischer Produete entstehen, so dass also nicht der Mangel an Blutgefässen, sondern jhre mehr oder minder betrabchlliche Veränderung - theils Verengerung des Lumens, theils Verlust der normalen Contractilität und Elasticitat - hier in Betracht zu ziehen sind.

Ein anderer, wie uns scheint, fiur die sichere Diagnose der in Rede stehenden Veranderungen wichtiger Punkt ist die ma agelnde Ausbildung der von Gegenbaur ${ }^{2}$ ) und Waldeyer ${ }^{2}$ ) beschriebenen Osteoblasten. Es ist in der That sehon bei schwächeren Vergrösseruggen auffallend, in wie geringer Menge diese eigenthimlichen, nicht zu verkennenden Bildungen in dem erkrankten Ossificationsgebiele vorhanden sind. Man muss lange suchen, bis man an den Rändern der Knocheubalken jene grossen vielgestaltigen Zellen antriff, welche in so naher Beziehung zum Verknockerungsprozesse steben. Wabrend sie am gesunden Knochen in einer fast contnuirlichen, epitbelahnlichen Lnge vorkommen, sieht man hier nur selten ganz vereinzelte grösseru Zellen, welche man als Osteoblasten ansprechen kann uod auch diese bei Woitem nich in der Ausbilung, wie bein normalen Verknobeherungsprozesse. Ws ist dreser Befund oin dureha

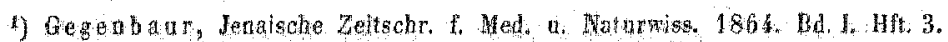

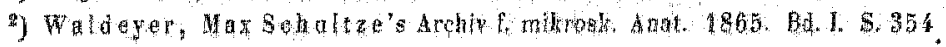


Dritten, welche dem Gange unserer Untersuchung nicht gefolgt waren, an unseren Präparaten auffiel. An Stelle der Osteoblasten finden sich mitunter jene kleinen Granulationszellen, welche in Folge dessen hart an den Knochen heran zu liegen kommen. In anderen Fällen wurden auch längere spindelförmige Elemente, in gleicher Flucht mit den Knochenbalken verlaufend, wahrgenommen; doch unterschieden sich diese immer deutlich von echten Osteoblasten.

Man muss nothgedrungen die Frage aufwerfen, wie unter diesen Verhältnissen der Verknöcherungsprozess vor sich geht, und man kommt zu dem Schlusse, dass unter Umständen auch jene, zu keiner besonderen Form ausgebildeten Granulationszellen im Stande seien, die Rolle der Knochenbildungszellen zn ubernehmen. Dass die Knochenbildung auf unregelmässigem Wege vor sich gehe, erhellt auch schon aus der genaueren Betrachtung der Knochenbalken, in denen man viele kleine und unregelmässig geformte Knochenkörperchen antrifft; auch deren Zahl und Lage weicht an manchen Stellen von dem gewohnten Verbalten ab.

Die von Wegner beschriebene Periostitis der Röhrenknochen haben wir ebenfalls und zwar in dem oben mitgetheilten Fall 3 in exquisitester Weise in der Näbe der Fpiphysengrenzen angetroffen. $\mathrm{Da}$ sie bei den übrigen Fällen mit wenigen hochgradigen epiphysären Veränderungen minder ausgebildet war oder selbst ganz fehlte, so möchten wir den Schluss zieben, dass diese verknöchernden PeriostAuflagerungen einem späteren resp. intensiveren Stadium des Prozesses entsprechen. -

Wegner hat den gesammten Prozess als einen entzündlichen unter der Bezeichnung „Osteochondritis“ aufgefasst. Bei den schwankenden Grenzen, welche das Gebiet der Entzündung, namentlich gegen die Gruppe der Granulationsueubildungen bat, liesse sich daruber rechten, ob jene Bezeichnung hier am Platze sei. In der einfachsten Weise wird der Prozess wohl als eine syphilitische Granulationsbildung aufgeführt. Die syphilitischen Producte treten, wie bekannt, in den verschiedenartigsten Formen zu Tage: entzündliche Vorgänge wechseln mit echten Neubildungsprozessen ab. Beide sind von demselben Virus bedingt; beide kommen an demselben Incividuum vor; zwischen beiden finden sich mannigfache unmerkliche Uebergänge. Dasselbe haben wir auch hier erfabren, indem wir bald mehr compacte, bald mehr eiteräbnliche Massen auftreten 
sahen, welche sich nach unserer Erfahrung allerdings immer noch hinreichend von gewöhnlichem Eiter unterschieden. Es scheint uns also nicht gerathen, hier obne Weiteres einen entzündlichen Prozess für alle diese Fälle anzunehmen. -

Wir lassen nunmehr eine kurze Aufzäblung unserer speciellen Befunde folgen. Lin Theil der untersuchten Kindesleichen wurde dem hiesigen pathologischen Institute Seitens der gynäkologisehen Klinik und Poliklinik überwiesen, deren Director, M. R. Prof. S piegelberg, die Benutzung der zugebörigen winischen Notizen fur die Veröffentlichung freundlichst gestattete.

Fall 1. 24. November 1869. Mutter vor 2 Jahren nachweislich constitutionell syphilitiseh. Keines mageres Kind. Abschürfungen und Geschwüre auf der Haut. Diffuse interstitielle Zellenwacherungen in den Lungen. Milaschwellung. Interstitielle Zellenwacherungen in der Leber und der Darnaschleimhaut. Schwelluag der Mesenterialdrüsen. Ecclymosen in den Kopflknochen und am Herzbeutel. 0edema glottidis. Rippen and belde Tibiae mit deutlichen syphilitischen Veränderungen. - Placenta nlcht nachgesehen.

Fall 9. 2. Janaar 1870. Neugeborenes Mädcber. Mutter im Verdacht constitutioneller Syphilis. Unfangreiche Ablösung der Epidermis an verschiedenen Stellen. Biffuse Verdickung der Gesichtshaut mit betrabhticher Wulstung der Lippen und Angenlider. Leichtes 0edem des Unterhautzellgewebes. Ascites und Oedem der Hirnläute, Hochgradige difuse Fleinzellige Granalationswueherung in der Thyutus, in beiden Lungen, der Leber, Milz, Nieren, Nebennieren, Magen and Darmwand, der Zunge und im weichen Gaumen, Atrophie der Thymus. Eiterige Bronchitis und Ecchymosen in den Lungen. Betrachtliche Vergrösserang der Leber und Milz, der Peyer'schen Plaques an der Cöcalklappe und der Mesenterialdrüsen. Zahlreiche Eechymosen der Pharyna- und Larynssehleimbant. An zablreichen untersuchten toochen: Rippen, Tibia, Fusswurzelknochen syphilltische Veränderungen. Placenta niebt untersucht.

Fall 3. 25. October 1870. Siebe oben.

Fall 4. 1. Januar 1871. Total macerirtes neugeborenes Kind. Placenta no r ma l. Die Eppliysen der langen Röhrenknochen üherall ron den Diaphysen gelöst. Bochgradige Vertnderungen an den Epiphysengrenzen An den Eingeweiden keinerlei Anomalien nachzoweisem.

Fall 19. Jonuar 18\%1. Macerirte Frübgeburt. Mutter aachweislich constitutionell syphilitisch. Syphilitische Veränderungen sämmtlicher Epiphysengreazen. (2. Stadium, Schweltung der Milz. Placenta nicht untersucht.

Fall 6. 10. März 1871. Fünf Wochen altes, seit vier Wochen an Pemphigus syphiliticus leidendes Kind, Wrtter mit circumscripten papalösea Syphiliden, Vater, yor 4 Jabren toficht, wit Pocriasie palmaris syph. behafet; erstere nie behandelt. Zwillingselo urt; der erte Zwilling war faultodt geboren und nicht untersacht worden. In den Lungen des zweiten Kindes knotige franulationswucherungen. 
Schwellung der Peyer'schen Plaques an der lleocoecalklappe (mikroskopisch: massenhafte kleinzellige, die Drüsen fast ganz verdeckende Wucherung in der Mucosa und Submucosa). Syphilitische Veränderungen an den Epiphysengrenzen der Tibia; die anderen Koochen sowie Placenta nicht untersucht.

Fall 7. 17. April 1871. Mutter ohne Verdacht auf Syphilis, Macerirtes neugeborenes Kind. Syphilitische Veränderungen an den Epiphysengrenzen der Röhrenlsnochen (im 2. Stadium). Placenta ohne nachweisbare Verănderungen.

Fall 8. 15. Mai 1871. Macerirter Knabe. Sypbilis der Mutter nicht nachweisbar. Ausgesprochene diffuse syphilitische Veränderung der Pla* centa ${ }^{1}$ ). Syphilitische Veränderungen der Epjphysengrenzen in 2. Stadium.

Fall 9. Juli 1871. Macerirter, etwa 26 bis 28 Wochen alter Fötus, Syphilis der Mutter nicht nachweisbar. Diffuse syphilitische Veränderungen der Placenta. Sypilitisehe Epiphysenaffection. Bedeutende Milaschwellung.

Fall 10. 1. August 1871. Vierteljähriger Knabe; gaboren 30. April, vom 18-31. Juli wegen papulöser und pustulöser Syphiliden mit ulceröser Destruction der rechten Ohrmuschel mit Sublimat-Chlornatrium (im Ganzen mit 0,03 Sublimat) behandelt. Mfntter seit 26 . Juni wegen Condylomata lata in Medication. Umfangreiche derbe interstitielle Granulationsheerde in beiden Lungenspitzen. Grosse derbe Nieren. UIceröse Perforation der linken Cornea. Ausgedebnte Verfettung der Neuroglia bes Gehirns. Unbedeutende makroskopische Veränderungen an den Epiphysengrenzen, welche sich mikroskopisch als durchaus conform dem syphilitischen Prozesse darstellen (Granulationszellen in den Markräumen, fast vollständiges Fehlen der Osteoblasten).

Fa ll 11. 11. August 18\%1. Marcerirtes männliches 8 monatliches hind. Syphilis von beiden Eltern in Abrede gestellt. Die Mutter gebar zuerst vier lebende Kinder nach einander, dann in den letzten 7 Jahren 5 faultodte Früchte. D iffuse Placentarsyphilis. Hochgradige syphilitische Veränderungen an den Epiphysen. grenzen der. Röbrenknochen.

Wenn der mangelnde Nacbweis constitutioneller Syphilis bei mehreren Müttern der vorstehend verzeichneten Kinder die ätiologische Begrindung der Knochenaffection der letzteren $\mathbf{z w e i f e l h a f t}$ erscheinen lassen könnte, so wird dieselbe nicht nur aufgewogen durch die völlig identischen Befunde in den Fällen 1, 2, 3, 5, 10, vor Allem aber im Falle 6, - Syphilis beider Eltern, Geburt von Zwillingen, deren einer faultodt, der andere neben ausgebreiteter Syphilis der Haut und Eingeweide eben diese Knochenaffection darbot, - sondern auch erläutert durch unsere nachfolgende letzte Beobachtung, in welcher wir zwar die Mutter intact, aber den $v_{a}$

1) Ueber die syphilitischea Verănderungen der Placenta wird demnächst Dr. Köbner im Verein mit Dr. Ernst Fränkel ausführliche Darstellungen geben. 
ter ${ }^{1}$ ) als vor der Zeugung syphilitisch constatirten. Der specifische Befund nicht blos in der Leber und in den Nebennieren, sondern auch in den Lungen dieses Kindes liefert zugleich einen Beitrag mehr gegen die Aufstellung von Bärensprung's (1. c. 111), dass sich die rom Vater ererbte Syphilis der Frucht nur in der Leber und den Nebennieren, die von der Mutter ererbte in den Lungen localisire. Die in den Lungen dieses Kindes vorgefundenen Heerde waren zwar bei Weitem nicht so zahlreich, noch auch so gross, wie jene, an Zabl wohl 30 betragenden, bis erbsengrossen, central schon erweichten Gummata in den Lungen des Kindes No. 3 ; sie gleichen aber in ihrer Structur vollkommen diesen, (vgl. bei Bärensprung Taf. 7 ) und überdies sahen wir auch bei von der Mutter aus sypbilitischen Kindern oft genug nur gleich kleine, zuweilen selbst gar keine Heerde. Umgekehrt bedarf es in Bezug auf die Existenz syphilitischer Veränderungen der Leber und Nebennieren bei Kindern dieser letzteren Categorie, als auf einen alluaglichen Befund, nur des Blickes auf unsere eigenen, sowie aul Hecker's, Wegner's and vielep Anderer Sectionsprotokole.

Fall 12. 4. November 1871. Neun Wochen altes, seit drei Wochen mit allgemeinen papulö§-squamösen Syphiliden, Coryza, Heiserkeil und Darmkatarrb behaftetes Kind, Multer, 29 Jabre alt, seil dem 19. Jahre eingegangene Ehe vor dem jetzigen kinde fünf gesunde zoboren, das fünfte, bis jetzt gesunde am 24. Jani 1868. Niemals hatte sie, and nirgends aulumen wir (am 28. October 1871) Zeichen oder Residnen von Syphilis wabr. Vater im December 1868 infieirt; Vleus penis, spăter Rachengeschwïre; bos örtlich behandelt, gegenwärtig symptomfrei. Jüngstes lind, wom 28. October mit Ung. Hydr. ciner. $(0,6$ p. die) eingerieben, zeigte am 2. November Perforation der linken Cornea und starb am 3. Noveraber.

Stamm und Extremitäten bedeckt yon zahllosen, scharfunden, sechsergrossen, weissgelbliehen trockenen Schuppen, unter welchen die Haut mabroskopisch normal erschein, Nase und Mondoffinung sowie die Dorsalseiten der Handgelenke bingegen bedeckt rod zusammengellessenen rothbräunlielien Borken, unter weichen oberlächliche Substanzererlusten Fettpolster reichlich. Linkes Auge ganz eingesunken, nleeröse Perforation der Connea so breit, wio dia Papille. In den Lappen der linker Lunge wehrere lobuläre Hepatisationsheerde tnit storker, hauptsächlich interalveolärrer Zelleninfiltration, welche anch einzelos Alveden erfült, die meisten comprimirt. In der enorm grossen, mit dem rechten lappen bis zur Crista ossis ilei dex, mit dera linken bis tef in das linke Bgochondrium retchenden Leber

1) Aetrologsol gleighe Beobachtungen nebst Sectionen finden sich bei fäbner. Whische a. exper. Nitbetl. a. d. Dermatal a, Syph. 1864 , hapitel: hereditäre Syph. F, 16 4. 17, Bärensprug, Heredit, Spplilis 1864, F. $33 \mathrm{u}_{4} 34$. 
interstitielle Granulationsheerde neben einzelnen fettig infiltrirten Partien. Milz nicht vergrössert, reich an Malpighi'schen Körperchen. Ueber der Bauhini'schen Klappe eine sebr gescluwellte Peyer'sebe Plaque, unterbalb jener weit hinab Schwellung der Solitärfollikel. Nieren intact. Nebennieren gross, in der braunrotberl Marksubstanz der rechten drei kleine, fast stecknadelkopfgrosse, weisse Einsprengungen. - Schädel dünn, regulär verknöchert, grosse Fontanelle ausgedehnt durch reichlichen Erguss im Subarachnoidealraum. Gehirnödem. Die Verbindungsstellen der Rippenknorpel mit dem knöchernen Theile der Rippen, sowie die 0ssificationsgrenzen der Epiphysen der Ulna, des Radius und Humerus sehen makroskopisch unverăndert aus, mikroskopisch fehlen daselbst die 0sieoblasten und finden sich die Markräume vollgepfropft von Granulationszellen, so dass ein von dem normalen mikroskopischen Verhalten des 0ssificationsgebietes ganz abweichendes Bild ercheint. Diesen letzteren, nur noch mikroskopisch scharf charakterisirten Grad der syphilitischen Veränderung haben wir sonach bei den beiden ältesten der von uns secirten Kinder, bei dem letzten, 9 Wochen alten und bei dem 12 Wochen alten (F. 10) angetroffen.

Die vorstehenden Beobachtungen, zusammengehạlten mit denjenigen von Valleix, Bargioni, Ranvier und Wegner lassen daribber keinen Zweifel, dass diese Erkrankung an den Epiphysen und Rippenknorpelgrenzen eine constante, intrauterin entstandene und von aer Behandlungsmethode der elterlichen Syphilis unabhängige ist. In letzterer Beziehung erinnern wir daran, dass ein Theil der Mütter gar nicht, ein Theil erst in den letzten 4 bis 6 Wochen vor der Entbindung (u. A. mit Sublimat-Chlornatrium), ein Theil endlich (mit Sublimat-Injectionen in der Berliner Charité) 3 bis 15 Monate vor der Conception behandelt worden war. So wenig daher die subcutane Sublimat-Injection syphilitischer Frauen vor diesem besten therapeutischen Prüfstein Stich hält, resp. etwa mehr leistet, als alle übrigen Behandlungsmethoden, so wenig steht diese Knochenaffection der Kinder in einem Zusammenhange mil irgend welcher, insbesondere mercuriellen Therapie der Eltern. Da sie sich nun auch bei keiner anderen allgemeinen Ernährungsstörung der letzteren findet, so hat sie in der That eine wesentliche diagnostische Bedeutung. Sie ist zu verwerthen erstens zur absoluten Diagnostik, z. B. bei macerirten Früchten, wo sehr häufig keine visceralen Veränderungen zu finden sind, und wo sie allein für die Aetiologie der Frühgeburt, bevor man noch eine Wiederbolung derselben abzuwarten braucht, sowie in zweiter Linie für die richtige Schätzung mancher dunkler Krankheitserscheinungen der Väter einen sicheren therapeutischen Anhaltspunkt gewälren kann; zweitens zur relativen 
Diagnose der bereditären gegenüber der acquirirten Syphilis infantum, wie Köbner dies bei einem in Folge von Syphilis e Vaccinatione gestorbenen Kinde 1. c. dargethan hat, - bei welchem die Ossificationsgrenzen der Rippen und Röhrenknochen entsprechend dem normalen makroskopischen Bilde auch ganz normales Markgewebe mit wobl entwickelten zablreichen Osteoblasten enthielten, und wie es in tödtlich ablaufenden Fällen auch bei anderweitigen Infectionsweisen kleiner Kinder, z. B. von dex Ammen her, fur den Gerichtsarzt ein maassgebliches Kriterium mehr abgeben dürte.

In Anhange an diese, fur congenitale Syphilis pathognomonische Knochenerkrankung wollen wir mit einer Beobachtung schliessen, welche für das Vorkommen auch von Rachitis bei älteren bereditär syphilitschen Kindern spricht. Leido fehit bei diesem Falle, der uns zu einer Zeit zuging, wo wir die Epiphysengrenzen noch nicht jedes Mal auch mikroskopisch genau uritersuchten, der mikroskopische Befund. Nichts desto weniger glauben wir ihn als an und für sich in mehrfacher Hinsicht beachtenswerth, und als einen vielleicht fiir künftige Untersuchungen aut dem Grenzgebiete zwischen Rachitis und hereditårer Knochensyphilis verwendbaren Beitrag, nicht übergehen zu sollen.

Vater zur Zeit der Zengung, Wotter bis über die Enthindung hinaus mit secundarer. Syphilis von uns beobachtet und vom Ende des 6. bis zur Mitte des 9. Schwangersehaftsmonates mercuriell (mit Quecksilberiodür und Sublimat) bebandelt. Kind, etwa 2 Wocben za früh am 9. October 1868 geboren, erkrankte in der 3. Woche an Erythemen der Palmae und Plantad pedum, welche sict binnen kurzem auf die ganzen Hände, Fässe, Ungebung des Wundes und aul die Nates ausbreiteten, an den letzteren Orten Rbagaden veranlassten and mit Desquamation, auch der Nagel, endeteb. Unter dem Gebrauche von Sublinat (innerlich, später in Bădern) heilte anch eir Pustelsyphilid am Kopfe bis gegen Weilunachten ab.

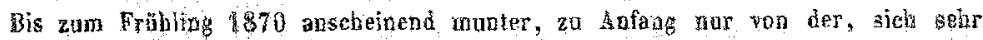
dưritig arnähreaden Mutter gesäugt, später uebenber avch mit "Slöpselbrei" gefüttert, litt Pat, öfte: an Darmkatarrh und belsam Milte Mai 1870 Convolsionen der Augenamsheln und Extremitäten sieben Tage hinter eirander, welche sich in Juni seltener wiederholten. Tod am 17. Juni. Section. 18. Junt 1870. Allgemeine Anamio. Chroniscbe Enteritis follicularis. Schwellung und diffuse Verfettung der Leber. Folliculampperplasie der Mili. Atrophie der Nebennieren. Braune Joduration der Lungen; Lungenödem. Mässiges 0edema cerebri, etwas weite Hirzventrikel mit leicht verdiuktem Ependym. In keinem Organe Heerderkrankangen.

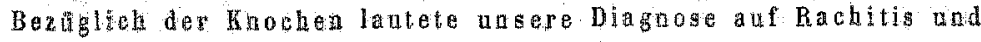


Craniotabes, daneben fand sich partielle Verknöcherung des Tentorium cerebellj. Der genauere Befund des Skelettes lautet: Umfang des Schädels dicht über den 0 hren $41 \mathrm{~cm}$., vom oberen Umfange einer Ohrmuschel bis zur anderen $23 \mathrm{Cr}$., von der Mitte der Glabella bis zur Höhe der Eminentia cruciata gemessen $24 \mathrm{~cm}$. Das Schädeldach hat also eine ziemlich rundliche Form, doch sticht der Hinterhauptstheil durch seine stärkere Entwickelung deutlich ab. Das Pericranium überall zart, lässt sich vom Stirnbein leicht, vom Schädel- und Hinterhauptsbein dagegen kaum ablösen. Grosse Fontanelle sehr weit. Die Stirnbeine überall gut entwickelt und gleichmässig fest verknöchert, namentlich stark ist die Knochensubstanz in der Gegend des unteren Endes der Kronennaht entwickelt, Schläfenbein und Hinterhauptsschuppe dagegen, sowie die Seitenwandbeine sind an den meisten Stellen fast papierdünn, nur einzelne, netzförmig mit einander zusammenhängende Zäge sind regulär verknöchert, dazwischen finden sich ganz dünne, bautartige Stellen ron Sechser- bis Fünfgroschengrösse. Dura mater haftet der Schädel- Innenfläche fest an. Längs der Pfeilnaht ist sie deutlich verdickt und mit querüber laufenden, sehr stark blutig injicirten Bindegewebsfäden belegt. Dieselben querziehenden verdickenden Fäden finden sicb in der linken Seite des grossen Hinterhauptsloches. Die obere Kante der Felsenbeine, namentlich des rechten, springt durch eine abnorme Verknöcherung des Tentorium crebelli fast $1 \mathrm{Cm}$. weit nach oben hervor, so dass sie an das Tentorium der Raubtbiere erinnert. Um das Torcular Herophili herum, sowie an den hinteren Abbängen beider Felsenbeine finden sich reichlicbe zarte, weiche osteoide Auflagerungen, die theils sehr bell, theils stark blutig gefärbt sind. Die Schädelnäthe, namentỉich zwischen den einzelnen Stücken der Hinterbaoptsschuppe sehr weit, die Nahthnorpel springen polsterartig hervor und haben in ihrer vächsten Umgebung eine Lage unverknöcherter osteoider Substanz. - Die Krorpel der Rippen deutlich angeschwollen; auf Durchschnitten derselben folgt auf den hyalinen linorpel eine sehr breite, hervorquellende, bläulich gefärbte Zone wuchernden Knorpelgewebes, dann eine andere ebenso breite Lage unverknöcherter osteogener Substanz, welche mit einzelnen Vorsprüngen mebr oder minder tief in die vorhergenannte Wucherungszone eingreift. Ein Durchschnitt durch den Kopf der rechten Tibia zeigt dasselbe Verhalten. Aeusserlich ist eine Schwellung der Gelenkenden wegen des starken Fettpolsters nicht wahrzunehmen.

Breslau, 6. December 1871. 